\title{
Neutrophil-derived JAML inhibits repair of intestinal epithelial injury during acute inflammation
}

\author{
DA Weber ${ }^{1,3}$, R Sumagin ${ }^{1,3}$, IC McCall ${ }^{1}$, Geoni $^{1}$, PA Neumann ${ }^{1}$, R Andargachew ${ }^{1}$, \\ JC Brazil ${ }^{1,2}$, O Medina-Contreras ${ }^{1,2}$, TL Denning ${ }^{1,2}$, A Nusrat ${ }^{1}$ and CA Parkos ${ }^{1,2}$
}

Neutrophil transepithelial migration (TEM) during acute inflammation is associated with mucosal injury. Using models of acute mucosal injury in vitro and in vivo, we describe a new mechanism by which neutrophils infiltrating the intestinal mucosa disrupt epithelial homeostasis. We report that junctional adhesion molecule-like protein (JAML) is cleaved from neutrophil surface by zinc metalloproteases during TEM. Neutrophil-derived soluble JAML binds to the epithelial tight junction protein coxsackie-adenovirus receptor (CAR) resulting in compromised barrier and inhibition of wound repair, through decreased epithelial proliferation. The deleterious effects of JAML on barrier and wound repair are reversed with an anti-JAML monoclonal antibody that inhibits JAML-CAR binding. JAML released from transmigrating neutrophils across inflamed epithelia may thus promote recruitment of leukocytes and aid in clearance of invading microorganisms. However, sustained release of JAML under pathologic conditions associated with persistence of large numbers of infiltrated neutrophils would compromise intestinal barrier and inhibit mucosal healing. Thus, targeting JAML-CAR interactions may improve mucosal healing responses under conditions of dysregulated neutrophil recruitment.

\section{INTRODUCTION}

Many inflammatory conditions of mucosal surfaces are characterized by epithelial wounds in association with robust infiltration of neutrophils, or polymorphonuclear leukocytes (PMN). Examples include Crohn's disease and ulcerative colitis where recruitment of PMN into the intestinal lumen parallels mucosal ulceration and patient symptoms. Conversely, resolution of PMN migration under these conditions is associated with mucosal healing and disease remission.

Sensing mucosal damage, PMN exit the microcirculation and migrate toward epithelial surfaces, driven by chemoattractants derived from invading organisms and resident cells that include bacterial peptides such as formyl-methionyl-leucyl-phenylalanine (fMLF) and chemokines such as interleukin- 8 and hepoxilin A3 ${ }^{1-3}$ PMN TEM is regulated by a complex series of leukocyte adhesive interactions with endothelial cells, matrix components, and epithelial cells. Receptor-ligand interactions that mediate migration of PMN across the epithelium are of particular importance given the association between disease symptoms and barrier dysfunction resulting from PMN transmigration.
Transmembrane receptors of the CTX (cortical thymocyte antigen of Xenopus) family of proteins expressed at intercellular junctions, including junctional adhesion molecules (JAMs), have been shown to regulate leukocyte interactions with endothelial and mucosal epithelial cells as well as epithelial cell homeostasis and barrier function. ${ }^{4-7}$ A closely related JAMlike molecule, $\mathrm{JAML}^{8}$ was reported to have a role in $\mathrm{PMN} \mathrm{TEM}^{9}$ through interaction with another CTX protein, coxsackieadenovirus receptor (CAR) localized to the tight junction (TJ) in epithelia and certain endothelia. ${ }^{9-11}$ Both JAML and CAR are type I glycoproteins containing two extracellular Ig-like domains, a single transmembrane helix, and a cytoplasmic tail with presumed signaling elements. ${ }^{12} \mathrm{CAR}$ is abundantly expressed in various epithelia and has been implicated in the regulation of epithelial permeability and cell adhesion to extracellular matrix, yet its function is incompletely understood. ${ }^{9-11}$ JAML expression is restricted to PMN, monocytes, and some T cells ${ }^{8,13,14}$ thus can mediate interactions with epithelial TJs through binding to CAR. In the skin, JAML was found to act as a costimulatory molecule for $\gamma \delta \mathrm{T}$ cells. JAML binding to CAR expressed on

\footnotetext{
${ }^{1}$ Epithelial Pathobiology and Mucosal Inflammation Unit, Department of Pathology and Laboratory Medicine, Emory University, Atlanta, Georgia, USA and ${ }^{2}$ Department of Pediatrics, Emory University, Atlanta, Georgia, USA. Correspondence: CA Parkos (cparkos@emory.edu)

${ }^{3}$ These authors contributed equally to this work.
} 
keratinocytes induced increased T-cell proliferation and production of cytokines and growth factors. ${ }^{14}$

In this study, we investigated the biology of JAML in PMN, and the functional consequences of JAML interactions with epithelial CAR in wound healing. We show that JAML is shed from PMN as a soluble molecule during TEM. We report that ligation of epithelial CAR by shed JAML impairs epithelial barrier function and inhibits mucosal wound healing. We discuss these findings in the context of epithelial injury associated with acute mucosal inflammation.

\section{RESULTS}

\section{Role of JAML/CAR interactions in PMN adhesion to intestinal epithelium}

CAR regulates epithelial barrier function while serving as a ligand for JAML on PMN. ${ }^{9-11}$ To investigate the functional biology of JAML-CAR interactions during PMN TEM, we generated monoclonal antibodies (mAbs) against the extracellular domain of human JAML (DW100 and DW216). These mAbs specifically recognized JAML on PMN, monocytes, and on differentiated promyelocytic cell lines HL-60 and PLB-985 (Supplementary Figure 1 online), and did not crossreact with other related CTX family members (Supplementary Table 1). Both mAbs bound the membrane distal domain (sJAML.D1), but not the membrane proximal domain (sJAML.D2) of JAML (Figure 1a). Since JAML binds to the membrane distal Ig-like domain of $\mathrm{CAR},{ }^{14}$ we tested whether these mAbs inhibited CAR-GST binding to immobilized sJAML-His. While, addition of DW100 prevented CAR-GST binding to JAML, DW216 had no effect (Figure 1b). Thus, DW100 and DW216 recognize two distinct epitopes on JAML-D1 domain and the DW100 epitope resides in close proximity/overlaps with the CAR binding site. Both DW100 (not shown) and DW216 (Figure 1c) immunoprecipitated a broad $\sim 55-65 \mathrm{kDa}$ protein band consistent with the size of full-length JAML from lysates of surface biotinylated differentiated HL60 and PLB-985 cells.

To confirm functional inhibition of JAML-CAR interactions by mAb DW100, we quantified PMN adhesion to monolayers of intestinal epithelial cells (T84 or Caco-2) expressing high levels of CAR under $\mathrm{Ca}^{2+}$-free conditions that served to open TJs and eliminate CD11b/CD18-dependent binding contributions. Addition of DW100 but not DW216 significantly inhibited PMN adhesion ( $35 \%)$ to both T84 and Caco-2 monolayers (Figure 1d). Furthermore, stable knockdown of CAR expression in Caco- 2 cells diminished adhesion of PMN to the level observed with addition of mAb DW100 (Figure 1d). Together, these findings demonstrate that JAML-CAR interactions mediate PMN adhesion to intestinal epithelium.

\section{PMN surface expression of JAML is lost during activation and TEM}

JAML-CAR interactions have been suggested to have a role in mediating PMN TEM, as soluble JAML and polyclonal JAML antiserum were reported to partially inhibit PMN TEM. ${ }^{9}$ However, we observed a relatively minor inhibitory effect of mAb DW100 on PMN TEM ( $\sim 15 \%)$, in contrast to major inhibition observed following inhibition of CD11b/CD18 (Supplementary Figure 2). We thus analyzed JAML expression on PMN following adhesion to epithelial cells and TEM. PMN adhesion to T84 monolayers resulted in a partial loss of surface JAML (1.5-fold decrease, Figure 2a,e), compared with control PMN in suspension. Intriguingly, near complete loss of JAML expression was observed after PMN migration across T84 or Caco-2 (not shown) monolayers (Figure 2b,e). In contrast, surface expression of CD11b/CD18 after TEM was increased. We further tested whether JAML expression on PMN was lost during PMN transmigration across monolayers of endothelial cells (human dermal microvascular endothelial cells) as has been observed for L-selectin. In contrast to the near complete loss observed during TEM, only a minor reduction in cell-surface JAML was observed after transendothelial migration (Figure 2c,e).

Since PMN TEM is linked to chemoattractant-stimulated activation, we examined whether PMN stimulation in suspension was sufficient to induce the loss of JAML or whether PMN contact with epithelial cells was required. PMN incubation with $10 \mathrm{nM}$ fMLF had no significant effect on JAML expression (not shown); however, treatment with $100 \mathrm{nM}$ fMLF resulted in a partial but significant loss of JAML expression (2.4-fold decrease, Figure 2d,e). Moreover, treatment with the potent activating agent phorbol myristate acetate (PMA) (200 nM) induced a near complete loss of cell-surface JAML from PMN, suggesting that the loss of JAML was both stimulus and dose dependent. In contrast, expression of CD18/CD11b was increased with both treatments indicative of PMN activation. Furthermore, loss of JAML upon activation with PMA was also observed in monocytes (Supplementary Figure 3). Interestingly, PMN adhesion to epithelial monolayers in the absence of an additional activating stimulus also resulted in decreased JAML expression $(\sim 1.5$-fold $)$, which was similar in magnitude to the decrease observed after migration across endothelial monolayers (Figure 2e). The loss of cell-surface JAML after PMN TEM was further confirmed by immunofluorescence staining (Figure 2f). These data suggest that loss of JAML in myelomonocytic cells is activation dependent and potentiated by contact with epithelial/ endothelial cells during the transmigration response.

We next examined whether JAML expression is also lost in vivo during PMN recruitment into inflamed murine intestine. In these experiments, PMN recruitment was induced by dextran sodium sulfate-mediated mucosal injury and JAML expression was analyzed on PMN isolated from colonic lamina propria (colon PMN) and compared with PMN freshly isolated from blood by flow cytometry using a hamster anti-murine JAML mAb. ${ }^{14}$ As observed with human PMN in-vitro, while JAML expression was detected on circulating murine PMN, it was lost on PMN isolated from the lamina propria of inflamed colons (Figure 2g, left panels). Given a recent report documenting JAML expression on the surface of $\gamma \delta \mathrm{T}$ cells, ${ }^{14}$ we compared the PMN JAML expression results with those on $\gamma \delta \mathrm{T}$ cells. JAML expression was unchanged on both circulating and colonic lamina propria $\gamma \delta \mathrm{T}$ cells (Figure $2 \mathrm{~g}$ ). 

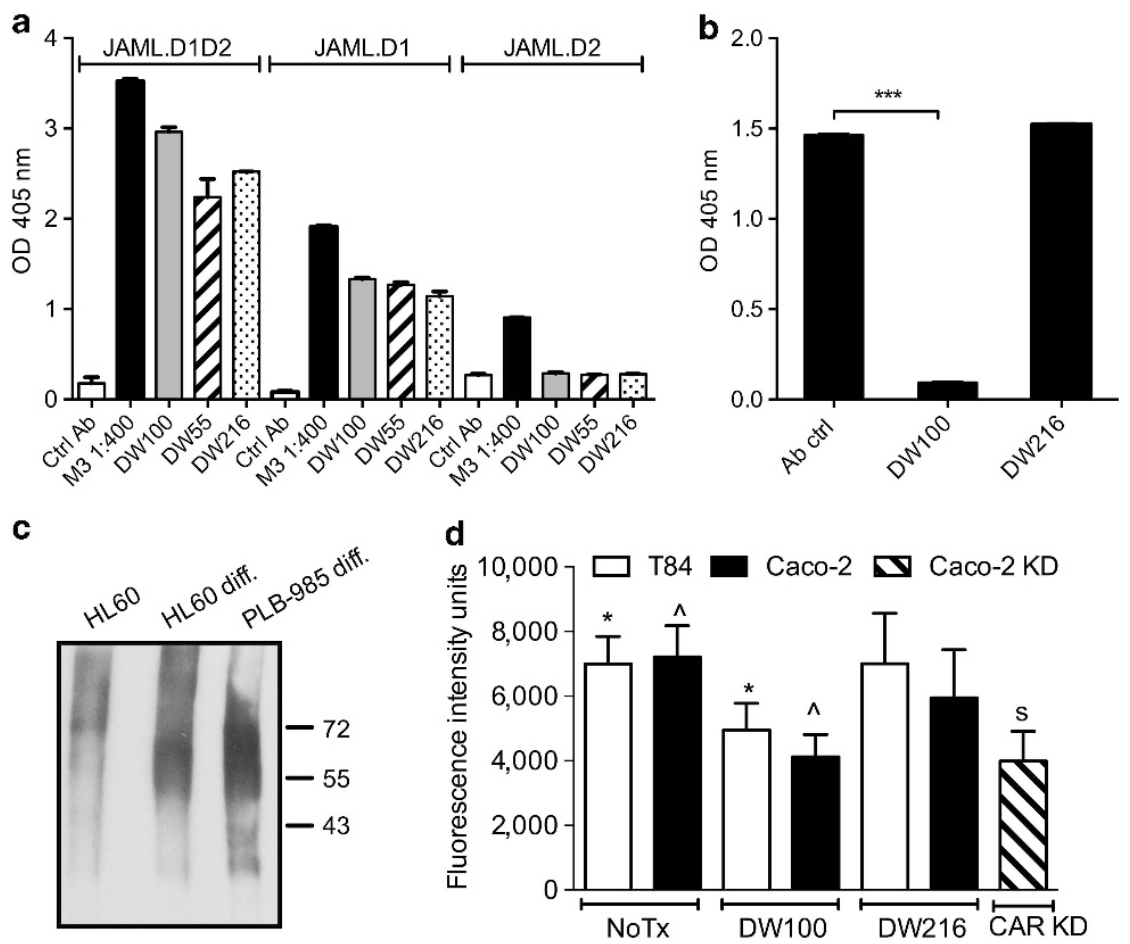

Figure 1 Characterization of an anti-human junctional adhesion molecule-like protein (JAML) monoclonal antibody (mAb) that inhibits JAML-CAR binding. (a) Anti-JAML mAbs were added to Immulon plates coated with soluble JAML (sJAML) (JAML.D1D2), membrane distal (sJAML.D1), or membrane proximal (sJAML.D2) domains. Antibody binding was detected using goat anti-mouse HRP. M3 represents JAML antiserum collected from the sJAML-His immunized mouse before fusion. Anti-Myc mAb (9E10) was used as a control. Both anti-JAML mAbs bound to the membrane distal domain (D1) of JAML. (b) mAbs to JAML $\left(10 \mu \mathrm{g} \mathrm{ml}^{-1}\right)$ were added to plates coated with SJAML followed by addition of CAR-GST (5 $\left.\mu \mathrm{g} \mathrm{ml}{ }^{-1}\right)$. CAR binding was detected by goat anti-GST-HRP. DW100 inhibited CAR binding to sJAML, but DW216 had no effect. ${ }^{* \star \star} P<0.01$ (significantly different). (c) JAML was immunoprecipitated from lysates of surface biotinylated cells, using DW216 mAb conjugated to sepharose beads. Western blot analysis using streptavidin-HRP revealed a broad protein band $(55-70 \mathrm{kDa}$ ) in lysates from differentiated cells. (d) A functional role for JAML-CAR binding interactions was tested in cell adhesion assays. Polymorphonuclear leukocytes (PMN) $\left(2.5 \times 10^{5}\right.$ per well) labeled with CellTracker Green were allowed to adhere to T84 (white bars), Caco-2 (black bars) or to stable Caco-2 lacking CAR (CAR KD) monolayers, in the absence of extracellular $\mathrm{Ca}^{2+}$ (to open tight junctions and eliminate contributions to CD11b/CD18-mediated adhesion) without (NoTx) or in the presence of DW100 or DW216 $\left(30 \mu \mathrm{g} \mathrm{ml}^{-1}\right)$. DW100 but not DW216 reduced adhesion of PMN to epithelial monolayers to the same levels observed after CAR knockdown. ${ }^{* / \wedge} P<0.05$ (significantly different from each other). ${ }^{\mathrm{S}} P<0.05$ (significantly different from control). $n=3$ independent experiments. CAR, coxsackie-adenovirus receptor.

We further confirmed that PMA-induced activation of lamina propria $\gamma \delta \mathrm{T}$ cells had no effect on surface expression of JAML (Supplementary Figure 4). Together, these findings suggest that JAML shedding during migration into the colon is specific to myelomonocytic cells.

\section{JAML is cleaved from the PMN surface during TEM by zinc-dependent metalloproteases}

Given the findings highlighted above, we hypothesized that the observed loss of cell-associated JAML during PMN activation was due to ectodomain shedding. To test this, mAbs DW100 and DW216 were used in a sandwich ELISA to capture and detect shed JAML ectodomains in cell-free supernatants. JAML was detected in supernatants from both PMA-activated PMN and differentiated PMN-like cell lines HL60 and PLB-985 (Figure 3a). Upon activation PMN are known to release membrane-bound microparticles/ectosomes from the cell surface, ${ }^{15}$ thus we measured the levels of JAML in supernatants from activated cells after high speed ultracentrifugation $(100 \mathrm{~K} g)$. No changes in the levels of JAML were observed, indicating that JAML is shed as a soluble molecule upon PMN activation (data not shown). Furthermore, the cleaved portion of JAML was immunoprecipitated from supernatants of biotinlabeled, PMA-stimulated PLB-985 cells and detected as a protein of $\sim 45 \mathrm{kDa}$ (Figure $3 \mathbf{b}$ ), consistent with the size of JAML extracellular domain (full size is $\sim 55-65 \mathrm{kDa}$, Figure 1c). To determine whether JAML release from activated leukocytes was dependent on cellular proteases, we stimulated differentiated PLB-985 cells with PMA in the presence or absence of a variety of protease inhibitors, and assayed for JAML in cell supernatants. A mixture of serine, cysteine, and several other protease inhibitors, as well as thrombin and serine protease inhibitors, AEBSF or $\mathrm{N}$-Ethyl maleimide failed to inhibit JAML shedding (data not shown). However, inhibition of $\mathrm{Zn}^{++}$-dependent metalloproteases with either phenanthroline or TAPI-2 $\left(\mathrm{TNF} \propto\right.$ protease inhibitor-2) ${ }^{16,17}$ significantly decreased cellular release of JAML after PMA activation (Figure 3c). This inhibition of JAML shedding was verified by fluorescent staining of differentiated PLB-985 cells with mAb DW216 (Figure 3d). While the possibility of further protease-dependent cleavage of JAML cannot be excluded, the above findings suggest that a fragment of JAML consisting of the majority of the 

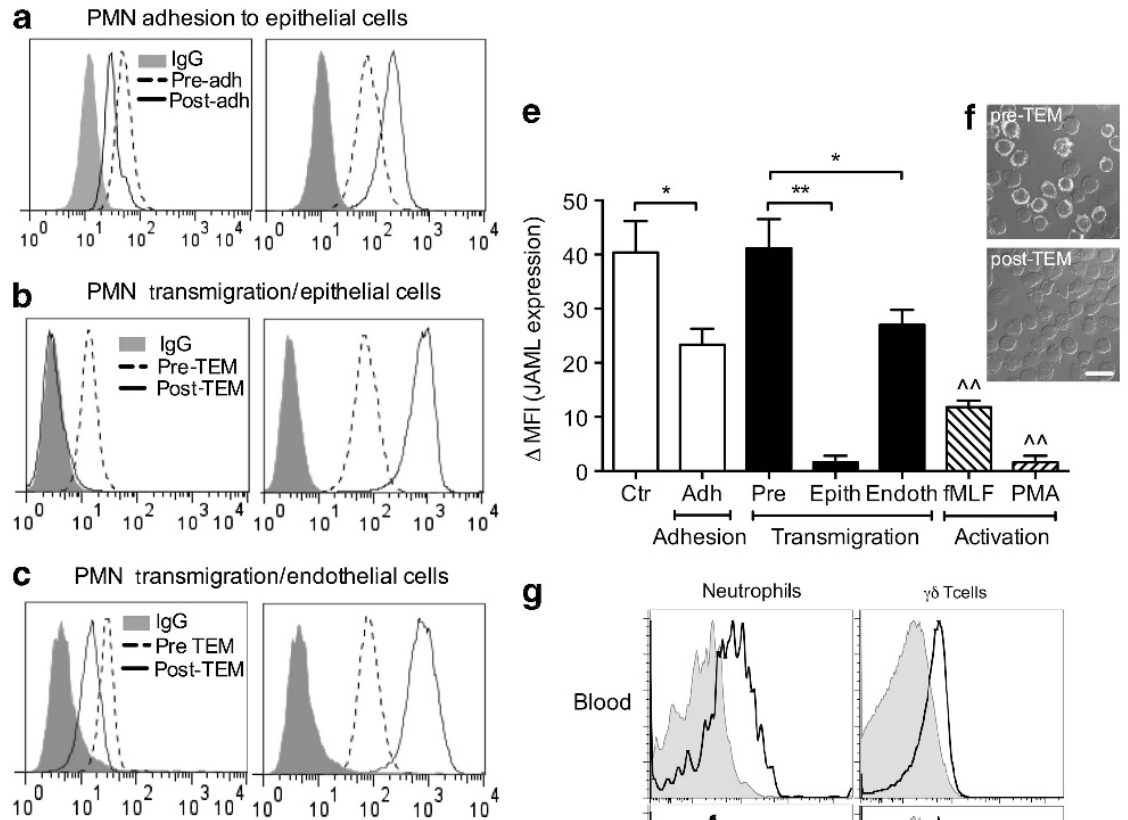

g
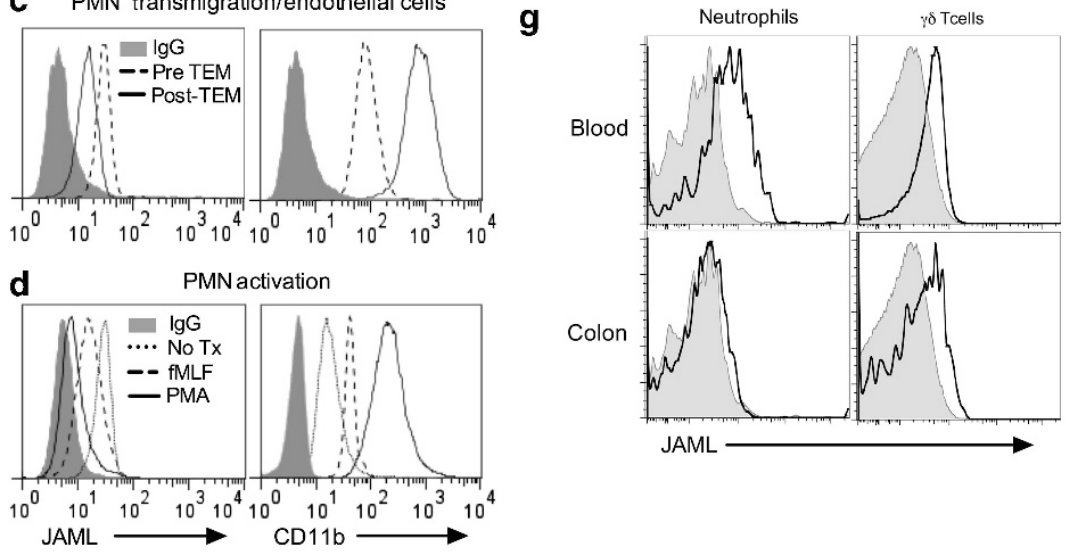

Figure 2 Junctional adhesion molecule-like protein (JAML) expression on polymorphonuclear leukocytes (PMN) surface is lost during transmigration in vitro and in vivo. (a-f) JAML and CD18/CD11b (as an index of PMN activation) expression on human PMN was examined by flow cytometry. (a) JAML expression on PMN after adhesion to epithelial cells was quantified by staining with DW216 monoclonal antibody $\left(\mathrm{mAb}^{2}\left(5 \mu \mathrm{g} \mathrm{ml}{ }^{-1}\right)\right.$. PMN were distinguished from epithelial cells by gating on CD18/CD11b-positive cells (CBRM 1/29, $5 \mu \mathrm{g} \mathrm{ml}^{-1}$ ). Adhesion to epithelium induced a partial loss of JAML. (b, c) PMN before or after migration across either T84 (b) or HDMVEC (c) monolayers were PFA fixed and stained for JAML and CD11b/CD18 as described above. Complete loss of surface JAML was observed after transepithelial migration, but only a partial loss after transendothelial migration. (d) JAML expression was examined after formyl-methionyl-leucyl-phenylalanine peptide (fMLF) (100 nM) and phorbol myristate acetate (PMA) $(200 \mathrm{nM})$ activation. fMLF activation resulted in a partial loss of JAML, however stronger activation with PMA triggered complete loss of JAML.

(e) Quantification of JAML expression, under the conditions specified above. ${ }^{*} P<0.05$, ${ }^{* *} P<0.001$ (significantly different). $M P<0.01$ (significantly different from control). $n=4$ independent experiments. (f) Representative immunofluorescence images of PMN before and after transepithelial migration. Scale bar is $10 \mu \mathrm{m}$. (g) JAML expression (line histograms) was examined in vivo, on murine circulating (upper panels) and colonic lamina propria (bottom panels) PMN and $\gamma \delta$ T cells, and compared with that on B cells (do not express JAML, filled histograms).

JAML expression was lost on PMN that infiltrated the colonic lamina propria, compared with circulating cells, but was unchanged on lamina propria $\gamma \delta \mathrm{T}$ cells. The micrographs are representative of three independent experiments.

extracellular domain is released from the surface of activated $\mathrm{PMN}$ in a $\mathrm{Zn}^{++}$metalloprotease-dependent manner.

\section{Soluble JAML binds to epithelial CAR and inhibits epithelial barrier function}

To examine whether JAML released from PMA-activated PMN was biologically active and was able to bind CAR, $\mathrm{CHO}-\mathrm{K} 1$ cells stably expressing CAR (CHO-CAR) were incubated with supernatants from PMA-stimulated PMN (200 nM) and JAML binding to CAR was confirmed by flow cytometry and confocal microscopy (Figure 4a,b). PMA treatment alone (200 nM) had no effect on the expression of CAR on CHO-CAR cells (not shown). To further investigate the biological effects of JAML ectodomains on epithelial barrier function in the absence of stimulating agents such as PMA, we generated soluble, recombinant JAML (sJAML) and confirmed its binding to CAR on CHO-CAR cells (Figure 4c, left panel). Similarly, we show the binding of soluble recombinant CAR to JAML stably expressed in $\mathrm{CHO}$ cells (CHO-JAML, Figure 4d, left panel). In both experimental setups, JAML-CAR binding was inhibited by DW100, but not by DW216 (Figure 4c,d, right panels), confirming the inhibitory function of DW100. Furthermore, using immunofluorescence labeling, we confirmed binding of sJAML to T84 epithelial cell monolayers, where it was found to colocalize with CAR (Figure 4e, upper panels). Addition of DW100 prevented these interactions (Figure 4e, bottom panels). Since CAR has been implicated in the regulation of TJ assembly and epithelial barrier function, ${ }^{18}$ we hypothesized that JAML released from migrating or activated PMN during inflammation may alter epithelial barrier function through binding to CAR. We examined the effects of sJAML on epithelial barrier recovery after $\mathrm{Ca}^{2+}$ switch assays by measuring transepithelial resistance and fluorescein isothiocyanate-labeled dextran flux $(3 \mathrm{kDa})$ as previously 


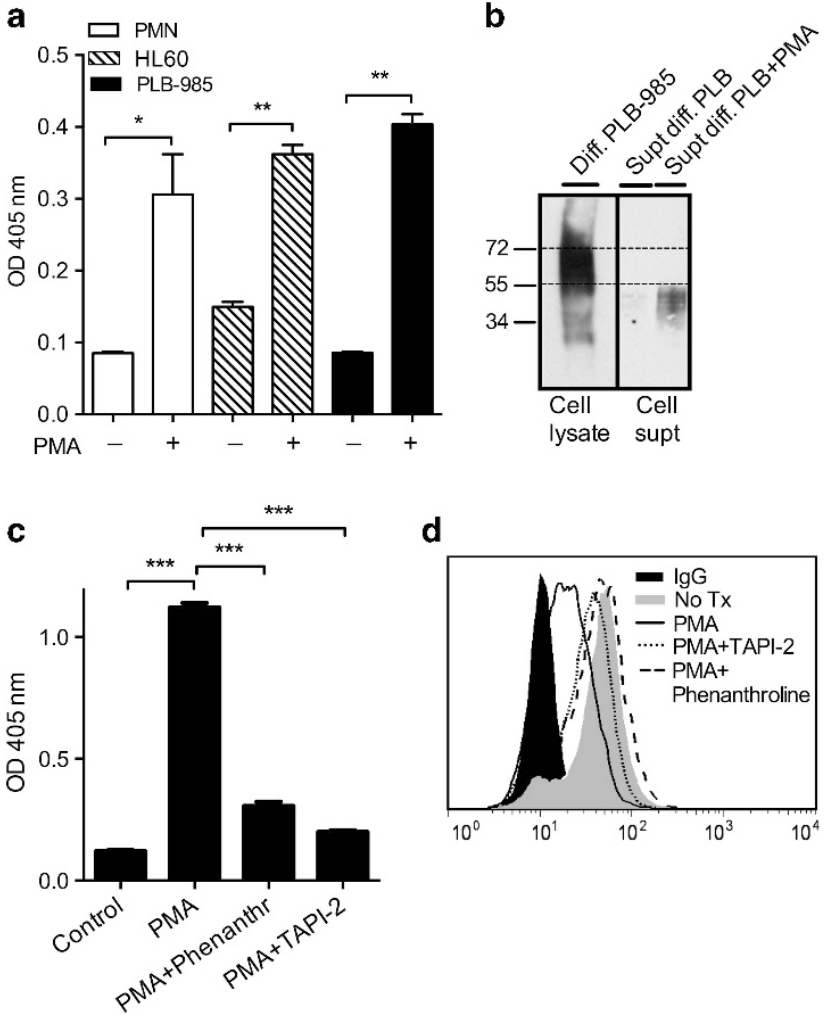

Figure 3 Junctional adhesion molecule-like protein (JAML) is cleaved from polymorphonuclear leukocytes (PMN) surface by $\mathrm{Zn}^{++}$-dependent metalloproteases. (a) Supernatants of phorbol myristate acetate (PMA) activated $(200 \mathrm{~nm}) \mathrm{PMN}\left(30 \times 10^{6}\right.$ cells per condition), differentiated HL60 and PLB-985 cells, after removal of microparticles (ultracentrifugation, $100 \mathrm{~K} \times \mathrm{g}, 30 \mathrm{~min}$ ) were analyzed for the presence of shed JAML using a capture ELISA as described in Methods. ${ }^{*} P<0.05,{ }^{* \star} P<0.001$ (significantly different). $n=4$ independent experiments. (b) JAML was immunoprecipitated using DW216-agarose bead conjugates from supernatants and total cell lysates of differentiated PLB-985 cells that were surface biotinylated and treated with or without PMA (200 nM). JAML immunoprecipitated from stimulated cell supernatants appears as a wide protein band between $\sim 45 \mathrm{kDa}$ suggesting that it contains a majority of the extracellular domain of JAML. (c) Capture ELISA of JAML shedding from differentiated PLB-985 after PMA treatment in the presence or absence of phenanthroline $(5 \mathrm{~mm})$ and TNF $\alpha$ protease inhibitor-2 (TAPI-2) $(1 \mu \mathrm{M})$, $\mathrm{Zn}^{++}$metalloprotease inhibitors. ${ }^{* \star *} P<0.001$ (significantly different). $n=4$ independent experiments. (d) Inhibition of JAML shedding from PLB-985 cells was confirmed by flow cytometry analysis of cells stained with DW216. In the presence of inhibitors, PMA activation had no effect on JAML expression.

described. ${ }^{19}$ Addition of sJAML, but not signal regulatory protein $\alpha(\operatorname{SIRP} \alpha)$ (binds a basolaterally expressed epithelial ligand $\left.\mathrm{CD} 47^{20}\right)$, significantly delayed recovery of barrier function as measured by transepithelial resistance (Figure 4f). Compromised epithelial barrier function in the presence of sJAML was still evident after $24 \mathrm{~h}$, as confirmed by 9-fold increase in dextran flux (Figure 4g). Importantly, inhibition of JAML-CAR binding with DW100, but not with DW216 (not shown) mAb reversed these effects.

\section{JAML-CAR interactions result in inhibition of intestinal epithelial wound healing}

PMN TEM is often associated with epithelial injury, we thus examined whether JAML-CAR binding influenced epithelial wound repair using in vitro scratch wound resealing assays. Addition of sJAML to scratch wounded monolayers significantly delayed wound closure ( $\sim 30 \%) 48$ and $72 \mathrm{~h}$ after injury (Figure 5a). Importantly, in the presence of DW100 but not DW216 sJAML-mediated inhibition of wound closure was reversed (Figure 5a).

It is accepted that epithelial cell migration mediates wound closure within the first $24 \mathrm{~h}$ of injury. ${ }^{21}$ However, we observed that sJAML had relatively minor inhibitory effects on wound closure within the first day suggesting that JAML binding to CAR affected cell proliferation rather than epithelial migration. Indeed using Edu incorporation assay, ${ }^{22}$ we found that addition of sJAML $(24 \mathrm{~h})$ to wounded T84 monolayers significantly decreased proliferation of cells at wound edges from $38.4 \pm 1.8 \%$ to $24.1 \pm 2.1 \%$ of Edu-positive cells (Figure $\mathbf{5 b}$ and representative images Figure 5c), however did not affect proliferation of cells remote from wounded areas (Supplementary Figure 5d). Consistent with the wound healing results, addition of DW100 but not DW216 (not shown) prevented the sJAML-dependent decrease in cell proliferation (Figure $\mathbf{5 b}$ ). These findings suggest that the inhibitory effect of sJAML is dependent on access to CAR at TJs, which is enhanced after loss of epithelial cell-cell contacts, as occurs after injury. We further observed that addition of sJAML to subconfluent epithelial cell monolayers also significantly decreased cell proliferation, which was reversed by DW100 but not by DW216 (Supplementary Figure 5e and f). These observations suggest that sJAML-mediated inhibitory effects are not restricted to wound edges.

Using TUNEL staining we found no effect of sJAML on epithelial cell apoptosis, thus ruling out the potential contribution for cell apoptosis in sJAML-mediated inhibition of epithelial wound healing (not shown). Consistent with the sJAML-mediated decrease in epithelial cell proliferation, addition of sJAML to wounded intestinal epithelial cell monolayers significantly attenuated wound-stimulated increases in phosphorylation of both c-Raf and Erk, which are key regulators of epithelial cell proliferation ${ }^{23}$ (Figure 5d). Confirming the specificity of these responses to sJAML, the observed effects were reversed in the presence of DW100 mAb (Supplementary Figure 5a-c). In contrast, sJAML had no effect on phosphorylation of Akt as well as $\beta$-catenin (not shown). Together, these findings suggest that ligation of CAR by sJAML inhibits epithelial cell proliferation leading to impaired epithelial wound healing.

\section{Ligation of CAR by SJAML or adenovirus protein Ad5 inhibits mucosal wound healing in vivo}

To extend the in vitro observations suggesting that binding of soluble JAML to CAR inhibits wound healing, we used a colonoscopic biopsy wound injury model in mice to examine the effects of CAR ligation on mucosal wound healing in vivo. We took advantage of another CAR ligand, adenoviral fiber knob protein 5 (Ad5) that has been shown previously to bind human and murine CAR with high affinity, ${ }^{14,24,25}$ and compete with JAML for CAR at the same binding site. ${ }^{14}$ Ad5, but not 
a

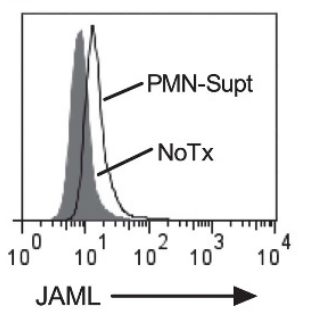

b

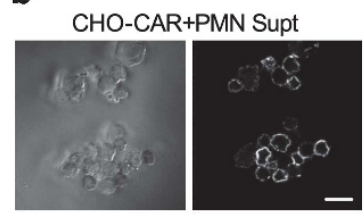

c

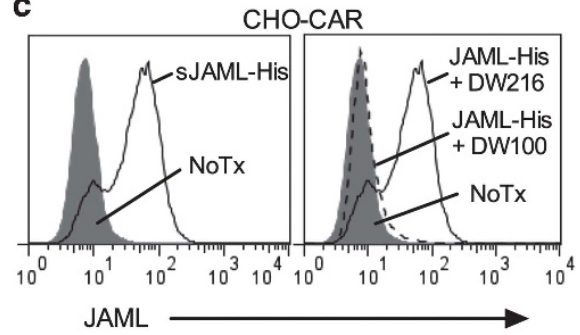

d
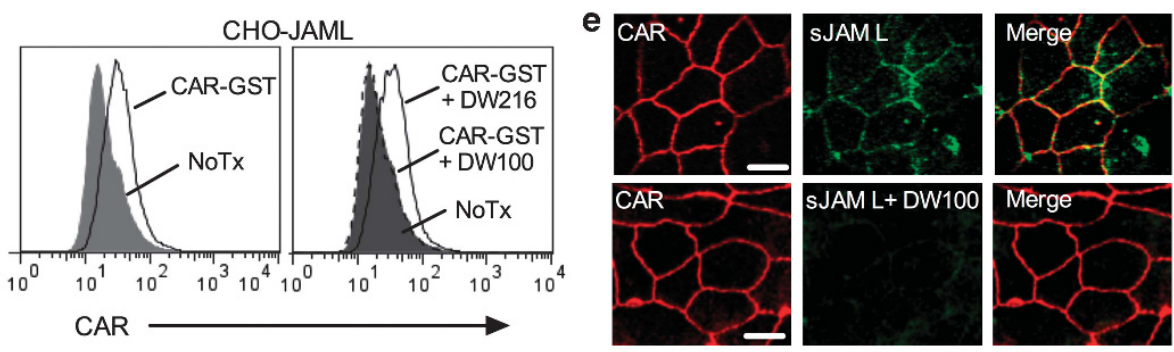

Merge
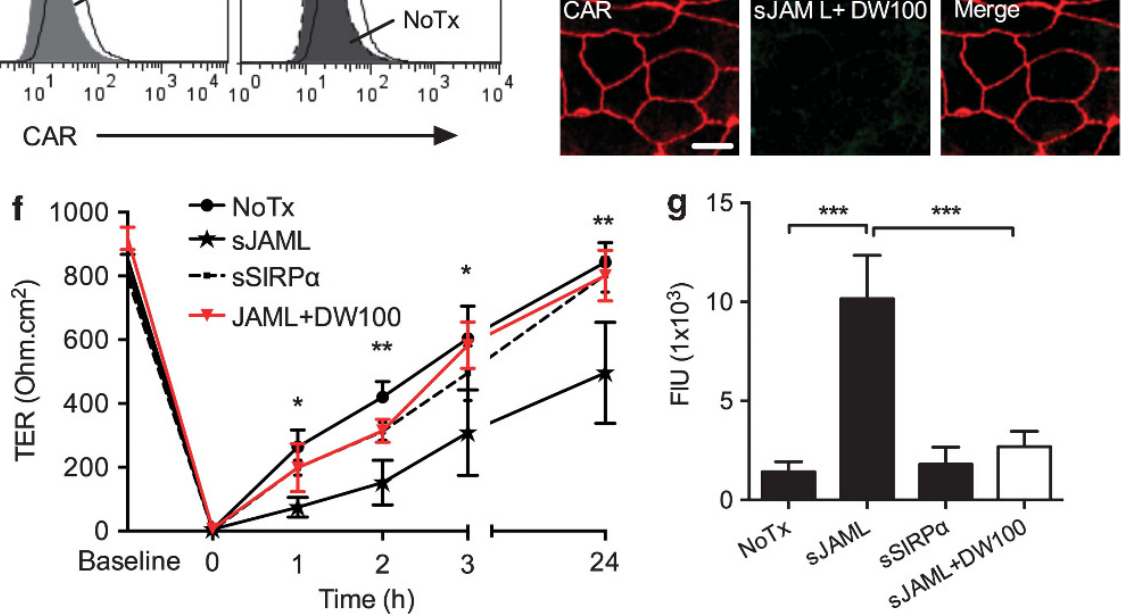

Figure 4 Shed junctional adhesion molecule-like protein (JAML) binds to coxsackie-adenovirus receptor (CAR) and inhibits epithelial barrier function. $(\mathbf{a}, \mathbf{b}) \mathrm{CHO}-\mathrm{CAR}$ stable transfectants were incubated with supernatants from phorbol myristate acetate (PMA) activated polymorphonuclear leukocytes $(\mathrm{PMN})\left(30 \times 10^{6}\right.$ per condition, $1 \mathrm{~h}$ on ice), and JAML binding to CAR was examined by flow cytometry (a) and confocal immunofluorescence microscopy (b). Scale bar is $20 \mu \mathrm{m}$. (c) CHO-CAR stable transfectants were incubated with soluble JAML-His alone (sJAML, $20 \mu \mathrm{g} \mathrm{ml}{ }^{-1}$ ) or in the presence of DW216 or DW100 monoclonal antibodies (mAbs) $\left(30 \mu \mathrm{g} \mathrm{ml}^{-1}\right.$, right panel). JAML binding to CAR was measured by flow cytometry and compared with untreated CHO-CAR (NoTx). (d) CHO-JAML stable transfectants were incubated with CAR-GST alone $\left(20 \mu \mathrm{g} \mathrm{ml}{ }^{-1}\right.$, left panel) or in the presence of DW216 or DW100 mAbs $\left(30 \mu \mathrm{g} \mathrm{ml}^{-1}\right.$, right panel) and binding was measured by flow cytometry. DW 100 but not DW216 prevented JAML-CAR interactions in CHO cells. Flow micrographs are representative of four independent experiments. (e) Representative immunofluorescence images confirm binding of SJAML to epithelial CAR. T84 monolayers, that were transiently preincubated in $\mathrm{Ca}^{2+}$-free buffer to increase access to tight junctions (TJs), were incubated with SJAML $\left(20 \mu \mathrm{g} \mathrm{ml}^{-1}\right)$ with/without DW100 mAb $\left(30 \mu \mathrm{g} \mathrm{ml}^{-1}\right)$ and stained for JAML (DW216, $\left.10 \mu \mathrm{g} \mathrm{ml}{ }^{-1}\right)$ and CAR $\left(\mathrm{H}-300,10 \mu \mathrm{g} \mathrm{ml}^{-1}\right)$. Colocalization of JAML and CAR is evident. Scale bar is $20 \mu \mathrm{m}$. (f) Barrier formation of T84 monolayers after a calcium switch assay was determined by transepithelial resistance (TER) measurements without (NoTx), or in the presence of control soluble signal regulatory protein $\alpha(\operatorname{SSIRP} \alpha)$ or SJAML $\left(20 \mu \mathrm{g} \mathrm{ml}^{-1}\right)$ with/without DW100 mAb $\left(30 \mu \mathrm{g} \mathrm{ml}^{-1}\right)$ at time points as indicated. ${ }^{*} P<0.05,{ }^{\star \star} P<0.005$ (significantly different from sJAML group). $n=4$ independent experiments. (g) Flux of fluorescein isothiocyanate-labeled dextran (3kDa) was measured after $24 \mathrm{~h}$ in a parallel experiment identical to that in $\mathbf{d}$. Addition of soluble JAML but not signal regulatory protein $\alpha(\mathrm{SIRP} \alpha)$ inhibits epithelial barrier recovery. The deleterious effects of SJAML on epithelial permeability were reversed with DW100 mAb. FIUs, fluorescence intensity units. ${ }^{* *} P<0.001$ (significantly different). $n=4$ independent experiments.

Ad11 (related adenoviral fiber knob control that does not bind to CAR) bound to immobilized human CAR and to $\mathrm{CHO}$ cells transfected with CAR, and to CAR-expressing murine intestinal epithelial cell line (CMT). Furthermore, Ad5 but not Ad11 inhibited binding of murine sJAML to CMT cells (Figure 6a) confirming that Ad5 binds to the same or overlapping epitope as JAML on CAR. Consistent with these results, addition of Ad5 but not Ad11 to both human (T84) and murine (CMT) intestinal epithelial monolayers significantly inhibited wound closure (Figure 6b,c) in a manner similar to inhibitory effects observed with sJAML. Importantly, intraperitoneal administration of sJAML and recombinant Ad5 in vivo (20 and $10 \mu \mathrm{g}$, respectively twice daily) significantly inhibited colonic mucosal wound healing (4 days after wounding) compared with Ad11 or phosphate-buffered saline (PBS) alone $(33 \pm 4.2 \%$ and $24.3 \pm 3.1 \%$ vs. $65.2 \pm 2.7$ and $66.7 \pm 3.2 \%$, respectively, Figure $6 \mathbf{d}$ ). In these experiments, superficial colonic mucosal wounds were induced in mice treated with Ad5, Ad11, or PBS using a mouse colonoscope as previously described. ${ }^{26}$ Healing of mucosal wounds was monitored 2 and 4 days post wounding by endoscopic imaging. Delayed wound closure was evident from histological analysis and whole mount preparations of wounded mucosa (Figure 6e, left and middle panels, respectively). Furthermore, 

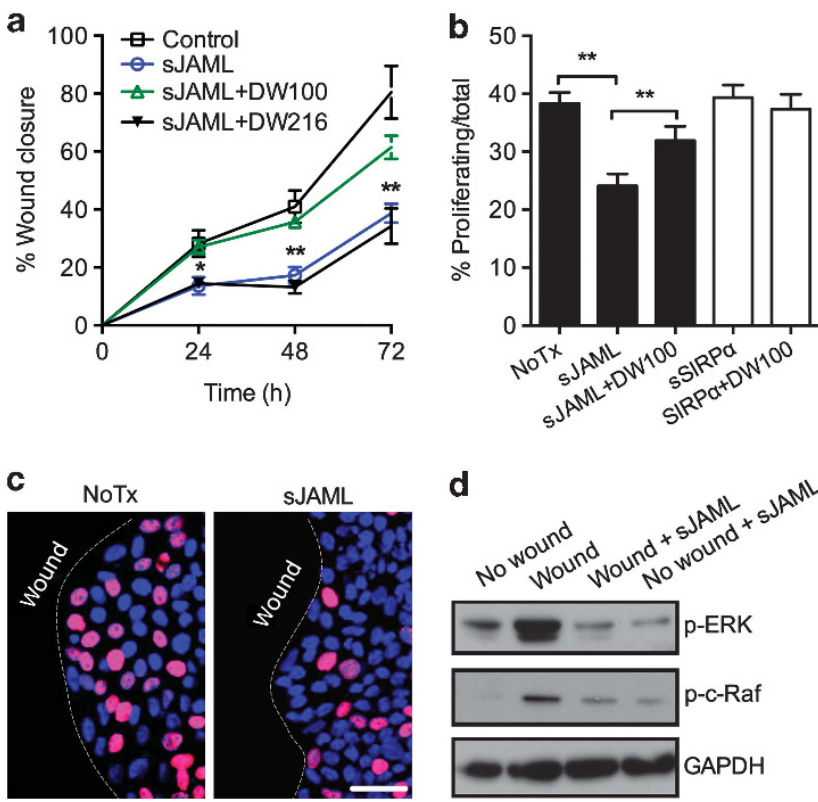

Figure 5 Ligation of coxsackie-adenovirus receptor (CAR) by soluble junctional adhesion molecule-like protein (SJAML) inhibits wound closure and decreases epithelial cell proliferation. (a) Intestinal epithelial monolayers grown to confluence were wounded by introduction of single linear scratch wounds. Wound closure was monitored over $72 \mathrm{~h}$ in the absence or presence of SJAML $\left(20 \mu \mathrm{g} \mathrm{ml}^{-1}\right)$ and DW100 (JAML neutralizing antibody, $50 \mu \mathrm{g} \mathrm{ml}^{-1}$ ). Addition of SJAML significantly inhibited wound closure. Importantly, in the presence of DW100, but not DW216, the inhibition was reversed. ${ }^{\star} P<0.05,{ }^{\star \star} P<0.005$ (significantly different from sJAML group). $n=4$ independent experiments. (b) Epithelial monolayers were wounded as described above and cell proliferation at the wound edge was assessed by EdU incorporation assay after $24 \mathrm{~h}$ in the presence of either SJAML or soluble signal regulatory protein $\alpha(\operatorname{SSIRP} \alpha)\left(30 \mu \mathrm{g} \mathrm{ml}^{-1}\right)$, with or without addition of DW100 monoclonal antibody (mAb). Data are presented as the percentage of proliferating cells (positively stained with EdU) relative to total cells per field. ${ }^{* *} P<0.01$ (significantly different). Cells were counted in seven randomly selected fields in four independent experiments. (c) Representative images depicting the decrease in proliferating cells at the wound edge in the presence of JAML. The scale bar is $50 \mu \mathrm{m}$. (d) SJAML inhibits wound closure by signaling through the ERK pathway. Western blots from lysates of wounded T84 monolayers demonstrating decreased $\mathrm{p}-\mathrm{ERK}$ and $\mathrm{p}-\mathrm{C}$-Raf signaling after addition of SJAML compared with untreated monolayers.

immunofluorescence labeling of frozen sections from harvested colonic mucosal wounds (day 4 after wounding) confirmed colocalization of Ad5 but not Ad11 with epithelial CAR in wounded areas (Figure 6e, right panels).

\section{Deleterious effects of PMN-derived JAML on epithelial wound repair are reversed by inhibition of JAML/CAR interactions}

We have shown that PMN activation, adhesion to, and migration across intestinal epithelium lead to shedding of JAML. Thus, we examined the direct effect of soluble JAML released from PMN on intestinal epithelial wound repair. PMN were applied to epithelial cell monolayers after introduction of linear scratch wounds and healing was assessed. Addition of PMN resulted in a significant inhibition of wound closure ( $\sim 40 \%$ ) after 48 and $72 \mathrm{~h}$ (Figure 7a). Importantly, anti-JAML mAb DW100 but not DW216 blocked a majority of PMN- dependent inhibitory effects on wound closure, confirming a major role for interactions between PMN-derived JAML with CAR in the regulation of intestinal epithelial wound healing (Figure 7a) and representative images Figure 7b). Together, these data indicate that JAML released from migrating leukocytes inhibits CAR-mediated regulation of intestinal epithelial wound healing under inflammatory conditions. Furthermore, inhibition of JAML-CAR interactions may be exploited therapeutically to improve healing responses under conditions of acute PMN recruitment to mucosal surfaces. From the above findings, we propose a model that highlights the role of shed JAML in the regulation of intestinal epithelial homeostasis under conditions of acute mucosal inflammation/ injury (Figure 8).

\section{DISCUSSION}

In the active phases of many mucosal inflammatory conditions, PMN TEM is associated with increased epithelial permeability, tissue damage, and patient symptoms. There are numerous reports detailing mechanisms by which high-density PMN trafficking across mucosal surfaces results in altered barrier function and epithelial homeostasis. During TEM, subepithelial PMN initiate contact-dependent epithelial signaling events that result in protease activated receptor-mediated alterations in barrier function. ${ }^{27}$ Additionally, proteases released by PMN, including elastase and cathepsin $\mathrm{G}$, have been shown to disrupt E-cadherin based epithelial cell-cell contacts ${ }^{28,29}$ while also inducing junctional discontinuities. ${ }^{30}$ Given these observations, it is not surprising that migration of large numbers of PMN across the epithelium results in sizeable discontinuities or ulcers/erosions. ${ }^{31}$ Here, we report a new mechanism by which JAML shed from activated PMN during TEM binds to the TJ protein CAR resulting in signaling events that alter epithelial function. Specifically, we demonstrate that sJAML interaction with CAR significantly impairs epithelial barrier function and wound healing. We speculate that during the early phases of an acute inflammatory response JAML shedding may serve to facilitate efficient recruitment of additional leukocytes to the sites on injury or infection by delaying barrier recovery. One could envision this mechanism acting to facilitate simultaneous influx of large numbers of PMN en-masse rather than recruitment of single PMN that would have to individually unzip junctions and squeeze between adjacent epithelial cells to get into the intestinal lumen. While the above mechanism would provide an efficient way to quickly clear enteric infections, resolution of the acute inflammatory response would be a necessary and vital component needed to restore mucosal homeostasis. Interestingly, failure to clear massive numbers of infiltrated PMN is observed in medically refractive ulcerative colitis, and under these pathologic conditions, sustained release of JAML could be linked to continued mucosal injury, ulceration, and patient symptoms.

Other distinguishing features of JAML include an unconventional extracellular structure resulting from rigid assembly of the Ig domains and an extended, flexible stalk. ${ }^{12}$ Such structure presumably facilitates unique interactions with CAR 
a
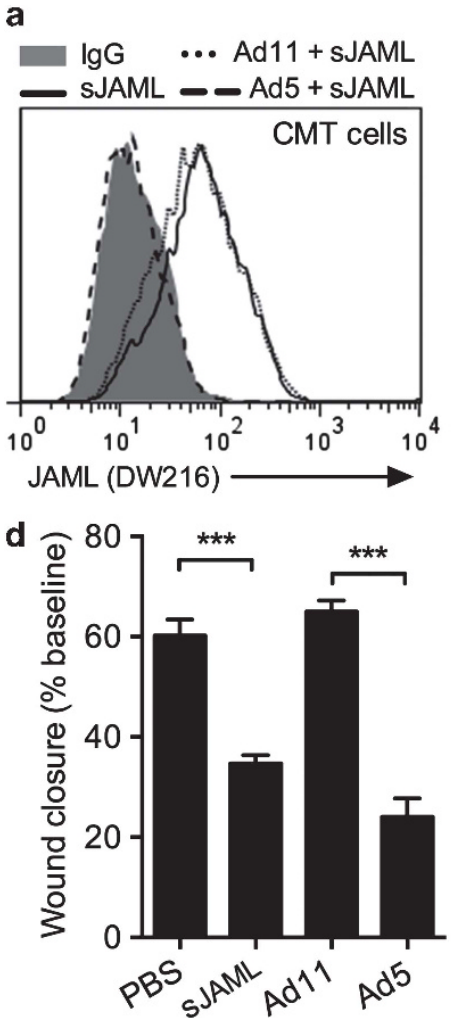
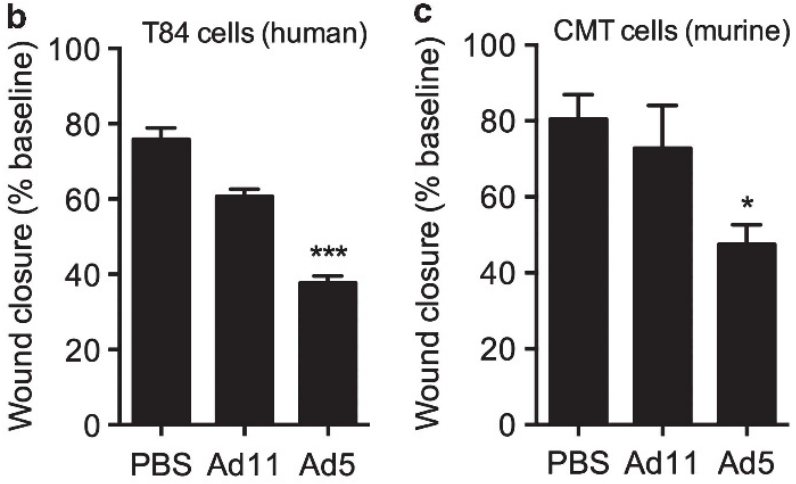

Figure 6 Coxsackie-adenovirus receptor (CAR) ligation by Ad5 or soluble junctional adhesion molecule-like protein (sJAML) inhibits epithelial wound repair in vitro and in vivo. (a) CAR expressing murine epithelial cells (CMT) were incubated with sJAML (20 $\left.\mu \mathrm{g} \mathrm{ml}^{-1}\right)$ in the presence of Ad5 (10 $\mu \mathrm{g} \mathrm{ml}^{-1}$ ) or Ad11 $\left(10 \mu \mathrm{g} \mathrm{ml}^{-1}\right)$. Addition of Ad5 but not Ad11prevented JAML binding. $n=4$ independent experiments. (b, c) Scratch wound assays were performed to examine the effects of CAR ligation by Ad5 $\left(10 \mu \mathrm{g} \mathrm{ml}^{-1}\right)$ and Ad11 $\left(10 \mu \mathrm{g} \mathrm{ml}^{-1}\right)$ on wound closure of T84 (human) and CMT epithelial cells. Addition of Ad5 but not Ad11 significantly decreased wound closure in both cell types. The data show quantification of wound area $72 \mathrm{~h}$ post wounding. ${ }^{\star} P<0.05,{ }^{\star \star \star} P<0.001$ (significantly different from phosphate-buffered saline (PBS)). $n=4$. (d, e) In vivo examination of the effects of CAR ligation by Ad5, Ad11, and murine sJAML on mucosal wound healing, using colonoscopic biopsy techniques. (d) Administration of sJAML (20 $\mu \mathrm{g}$ per $100 \mu \mathrm{l}$, intraperitoneally (IP) twice daily) and Ad5 but not Ad11 (10 $\mu \mathrm{g}$ per $100 \mu \mathrm{l}$, IP twice daily) or PBS significantly delayed wound closure. The data show quantification of wound areas 4 days post wounding. ${ }^{\star \star \star} P<0.005$ (double blinded study, 5 mice per group). $n=3$ independent experiments.

(e) Hematoxylin/eosin-stained wound sections (left panels) and representative images of excised whole mounts of mucosal wounds (middle panels, the bar is $200 \mu \mathrm{m}$ ) at day 4 post wounding demonstrate near complete healing in the presence of Ad11 (upper panels) but not Ad5 (bottom panels). Ad5 colocalization (yellow) with CAR in wounded regions was confirmed by immunofluorescence staining (CAR, red and His-tagged Ad5 and Ad11, green).

a

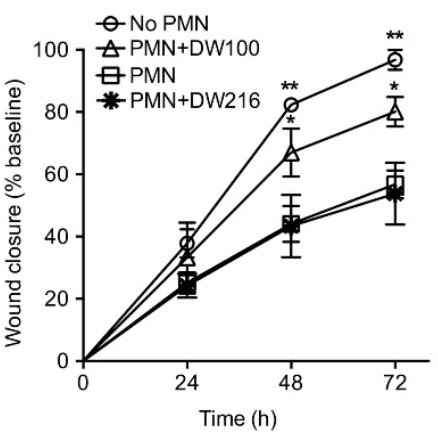

b

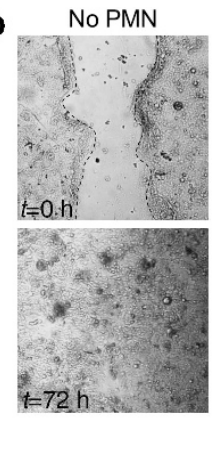

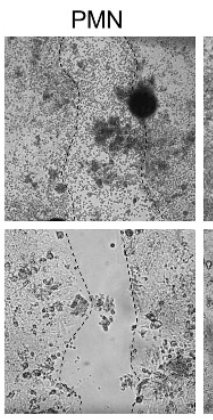

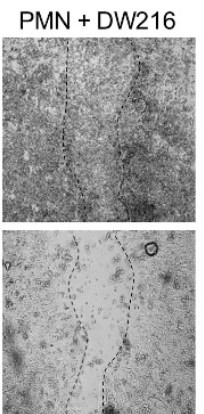

$\mathrm{PMN}+\mathrm{DW} 100$

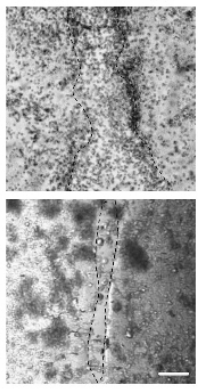

Figure 7 Inhibitory effects of polymorphonuclear leukocytes (PMN) on epithelial wound repair are significantly attenuated by blocking CAR-JAML interactions. (a) T84 monolayers grown to confluence were wounded by introduction of single linear scratch wounds. Wound closure was monitored over $72 \mathrm{~h}$ in the absence or presence of PMN $\left(1 \times 10^{6}\right.$ per well), and/or DW100 (JAML neutralizing antibody, $\left.30 \mu \mathrm{g} \mathrm{ml}{ }^{-1}\right)$ and DW216 $\left(30 \mu \mathrm{g} \mathrm{ml}^{-1}\right)$ antibodies. The presence of PMN significantly inhibited wound closure. Importantly, addition of DW100 but not DW216 significantly reversed PMN-mediated inhibition of wound closure. ${ }^{*} P<0.05$, ${ }^{*} P<0.005$ (significantly different from PMN group). $n=4$ independent experiments. (b) Representative images of epithelial monolayers immediately after wounding and addition of PMN ( $t=0$, upper panels), and after $72 \mathrm{~h}(t=72$, bottom panels) demonstrating inhibitory effects of PMN on wound healing, and reversal of inhibition with DW100 monoclonal antibody (mAb). The scale bar is $25 \mu \mathrm{m}$. JAML, junctional adhesion molecule-like protein; CAR, coxsackie-adenovirus receptor. 


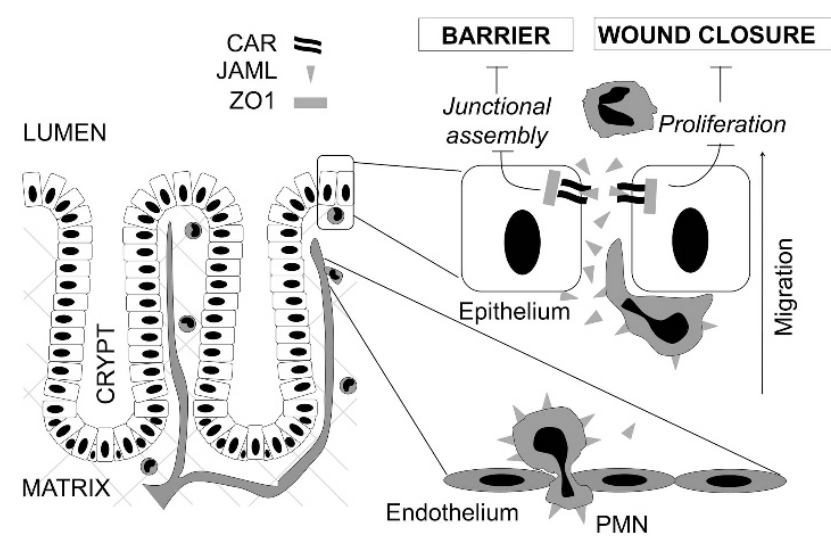

Figure 8 Model depicting a paracrine role of junctional adhesion molecule-like protein (JAML) in inhibition of intestinal epithelial restitution during acute inflammation. Acute polymorphonuclear leukocyte (PMN) recruitment to mucosal surfaces in response to microbes or injury occurs in several steps. First PMN exit the microcirculation by migrating across the vascular endothelium where JAML is mostly retained on the PMN cell surface. As PMN migrate across the epithelium, JAML is cleaved from the cell surface where it binds to coxsackie-adenovirus receptor (CAR) at tight junctions. Soluble JAML binding to CAR results in disruption of CARmediated signaling events and inhibits tight junction reassembly/barrier formation secondary to PMN transmigration. Furthermore, soluble JAML inhibits CAR-dependent epithelial proliferation at wounds in close proximity to migrating/activated PMN resulting in delayed mucosal healing. Thus, release of JAML would provide an efficient proinflammatory mechanism to facilitate recruitment of additional leukocytes and thus aid in clearance of invading microorganisms. However, sustained release of JAML under pathologic conditions associated with persistence of large numbers of infiltrated PMN would inhibit mucosal healing. ZO1, zonula occludens- 1 .

on the surface of epithelial cells. Ligand binding is mediated by the membrane distal Ig-like domain of JAML (JAML.D1) and, as shown in this report, is completely inhibited by a D1 binding mAb (DW100) (Figure 1). By contrast, another anti-JAML D1 mAb DW216 did not affect JAML binding to CAR.

Using anti-JAML mAbs, we report that JAML expression on PMN and monocytes is decreased/lost after activation with fMLF and PMA and that the loss of JAML correlates with the degree of PMN activation and nature of the given stimulus. Interestingly, using polyclonal antiserum raised against sJAML-Fc, we previously reported increased staining in activated PMN. ${ }^{9}$ However, while producing anti-JAML specific mAbs, we identified antibodies with crossreactivity to other JAM proteins. Thus, binding of polyclonal anti-JAML antiserum to related molecules may have contributed to such findings. As shown in Supplementary Table 1, the anti-JAML mAbs in this report do not crossreact with other related JAM proteins. Additionally, in the current study, we report dose and agonist-dependent shedding of JAML that requires zincdependent metalloprotease activity in PMN. Thus, priming of PMN during isolation coupled with differences in activation of metalloproteases may affect cell-surface JAML expression and PMN function. Indeed, addition of the zinc-dependent metalloprotease inhibitor TAPI-2 to suspensions of PMN not only inhibits shedding of JAML but also significantly increases JAML-dependent PMN TEM that is specifically blocked by DW100. Given this observation one might thus ask whether
JAML shedding during PMN activation may act as a brake and reduce TEM. In the case of luminal antigen/bacterial leakage into the lamina propria, it might be advantageous for recruited PMN to be retained in the subepithelial space to clear microbes locally, rather than migrating into the lumen. However, we have shown that $\mathrm{CD} 11 \mathrm{~b} / \mathrm{CD} 18$ is by far the dominant regulator of PMN-epithelial adhesion/transmigration and that blockage of JAML under conditions supporting CD11b/CD18-mediated migration has only minor effects on PMN TEM. Interpretation of experiments testing whether JAML shedding is directly associated with decreased PMN TEM is confounded by other factors secondary to PMN activation, so contributions from JAML loss in this context remain unclear. Alternatively, it is possible that conditions resulting in diminished zincdependent metalloproteinase activity could enhance PMN migration and further augment the inflammatory response at the level of the epithelium.

Zinc metalloproteases mediate cleavage of L-selectin from the PMN surface ${ }^{32}$ during early PMN rolling on vascular endothelium. ${ }^{33}$ In contrast, JAML was mostly retained on PMN surface following transendothelial migration and was cleaved during TEM. Perhaps such activation is linked to the level of CAR expression that has been shown to be low and variable in different endothelia, ${ }^{34,35}$ but highly expressed on intestinal and other types of epithelial cells. Given the strong association of JAML cleavage with TEM, the shed ectodomains would be in intimate contact with epithelial cells, and by binding to CAR, could affect epithelial function in a paracrine manner. Such findings also likely apply to monocytes as we and others ${ }^{13}$ have observed significant levels of JAML expression on monocytes, and our studies show that monocyte-associated JAML is also lost upon activation. Given that monocytes are also critical in the innate immune response and represent early arrivers to sites of mucosal inflammation, one can envision these innate immune cells as an additional source of released JAML ectodomains that could amplify PMN-mediated effects on epithelial function.

In this report, we demonstrate that the shed/soluble JAML ectodomain inhibits epithelial barrier function and wound healing. It is well established that epithelial wound healing involves enhanced directional polarization and migration of cells at the leading edge, followed by increased cell proliferation at later time points. ${ }^{21}$ Our results suggest that interactions between JAML and CAR delay intestinal epithelial wound closure by inhibiting cell proliferation. After probing candidate signaling pathways, we found that sJAML inhibition of wound closure is mediated by decreased ERK signaling, a key pathway known to be involved in the regulation of epithelial cell proliferation. ${ }^{23,36}$

A recent study showed beneficial effects of JAML-CAR interactions on dermal wound healing mediated by activation of JAML on $\gamma \delta \mathrm{T}$ cells in mouse skin. In that study, it was shown that $\gamma \delta \mathrm{T}$ cell interactions with keratinocytes mediated through JAML-CAR binding resulted in JAML-mediated T-cell proliferation along with production of T cell-derived cytokines and keratinocyte growth factor, which proved to enhance dermal wound closure. ${ }^{14}$ These observations highlight signaling events 
secondary to JAML-CAR binding that are cell type and context specific. While ligation of JAML expressed on T cells in the skin results in T cell-derived pro-restitutive responses, in this study, we show that JAML-mediated ligation of CAR expressed on epithelial cells in the intestine results in epithelial-derived inhibitory restitutive responses. While the role of JAML in PMN function is not clear, it is most certainly distinct from that reported in $\mathrm{T}$ cells. One notable difference highlighted in Figure $2 \mathrm{~g}$ of this study demonstrates no change in JAML expression on $\gamma \delta \mathrm{T}$ cells isolated from the colonic lamina propria during colitis, or following direct activation with PMA (Supplementary Figure 4), which has been previously shown to activate $\mathrm{Zn}^{++}$-dependent metalloproteases. ${ }^{37}$ These findings suggest that, unlike PMN and monocytes, T cells do not shed JAML upon infiltration of the colonic mucosa. During acute mucosal inflammation, one could envision significant amounts of soluble JAML released in the immediate vicinity of high-density PMN migration. Ligation of epithelial CAR under these circumstances would likely have a pro-inflammatory effect. Similarly, the findings in this report raise the question of whether PMN-derived JAML might have a role in poor healing of mucosal ulcers associated with massive PMN infiltration in conditions such as ulcerative colitis.

Given known functions of related CTX family members, it is reasonable to propose that CAR ligation by JAML mediates outside-in signaling to affect multiple cellular functions. For example, studies on a closely related TJ protein JAM-A have revealed that homodimerization in the membrane distal D1 domain results in close apposition of cytoplasmic scaffold molecules to facilitate signaling events. JAM-A dimerization enhances Rap1-mediated effects on cell migration via control of cell-surface $\beta 1$-integrin levels. ${ }^{38,39}$ JAM-A dimerization has also been shown to regulate epithelial cell proliferation by Akt, $\beta$-catenin signaling pathways. ${ }^{6}$ CAR that is expressed at epithelial TJs colocalizes with the cytoplasmic plaque protein zonula occludens- $1 .{ }^{11}$ While CAR is mainly recognized as the receptor that mediates coxsackie-adenovirus attachment, ${ }^{40,41}$ like JAM-A, it has also been reported to have a role in the regulation of epithelial permeability and TJ reassembly. Addition of soluble CAR to intestinal epithelial monolayers after $\mathrm{Ca}^{2+}$ switch assay has been shown to inhibit TJ reassembly. ${ }^{11}$ Like JAM-A, CAR has been reported to dimerize in $\mathrm{cis}^{42}$ in the D1 domain which is also a presumed binding domain for JAML. ${ }^{12}$ It is thus possible that JAML binding may inhibit CAR-CAR dimerization, altering downstream signaling events.

In summary, we present a model in Figure 8 highlighting acute PMN recruitment by mucosal inflammatory cues resulting in shedding of a majority of cell-surface JAML during TEM. Shed JAML molecules bind TJ-associated CAR and alter signaling events resulting in perturbed barrier function and inhibition of epithelial cell proliferation leading to impaired wound closure. While such responses may serve to enhance the early phases of acute mucosal inflammatory responses, they may also be responsible for poorly healing mucosal ulcers associated with massive PMN infiltration in conditions such as ulcerative colitis.
Development of novel therapeutics that specifically block JAML-CAR receptor-ligand interactions in the intestine may have a significant value in promoting barrier function and epithelial wound closure under conditions associated with pathologic accumulation of neutrophils in the mucosa.

\section{METHODS}

Cells. HL60, wild-type Chinese hamster ovary cells (CHO), and HEK293T human embryonic kidney cell line were obtained from ATCC (Rockville, MD). HL60 and PLB- $985^{43,44}$ were passaged in RPMI containing 20\% heat inactivated FBS (Atlanta Biologicals, Atlanta, GA) with supplements and differentiated as previously described. ${ }^{44} \mathrm{CHO}-\mathrm{K} 1$, HEK293T cells, Caco-2, and T84 epithelial cell lines were cultured as described. ${ }^{45}$ Human dermal microvascular endothelial cells (passages 4-9) were a kind gift from Nancy Louis. Human blood was drawn and handled according to protocols for the protection of human subjects, as approved by the Emory University Hospital Institutional Review Board, and in accordance with the Declaration of Helsinki (2000). PMN were isolated by density gradient centrifugation. $^{45}$

Generation of recombinant proteins. All plasmid constructs were generated by the Custom Cloning Center Facility at Emory University and sequences confirmed. Human JAML (GenBank: AJ515553.2) was cloned in pcDNA3.0 (Invitrogen Life Technologies, Grand Island, NY) with C-terminal His tags or 6XMyc or rabbit-Fc. ${ }^{20}$ The extracellular domains of JAML were tagged after residue $\mathrm{Leu}^{259}$ for JAML.D1D2-His (sJAML), and Pro ${ }^{140}$ for sJAML.D1. CHO or HEK293 T cells were transfected using polyethylenimine (PEI), and stable clones selected with G418/Hygromycin B appropriately. Soluble His-tagged proteins were purified on Ni-NTA agarose beads (Qiagen, Valencia, CA). Soluble CAR-GST construct has been described. ${ }^{9}$ Full-length CAR and JAML constructs were expressed in $\mathrm{CHO}$ cells (CHO-CAR or CHO-JAML) and expression assessed by flow cytometry. Adenofiber knob proteins Ad5-His (Ad5) and Ad11-His (Ad11) were expressed in E. coli from respective constructs (a kind gift of G. Nemerow, Scripps Institute, La Jolla, CA). Soluble murine JAML-mFc construct was cloned into pVL1393 (Invitrogen, Carlsbad, CA), expressed in the Baculovirus system (Invitrogen) and purified on Protein A Sepharose.

Antibodies and reagents. $\mathrm{RmcB}$ and $9 \mathrm{E} 10$ were purified from hybridomas. Anti-CD11b/CD18 (CBRM1/29) was previously described. ${ }^{46}$ Anti-human CAR (H-300) was from Santa Cruz (Santa Cruz, CA). Murine PMN and $\gamma \delta$ T cells: CD3€eFluor450 (eBio500A2), CD45-PerCP-Cy5.5 (RM4-5), TCR $\gamma \delta$-fluorescein isothiocyanate (GL3), Gr1-APC-eFluor780 (RB6-8C5), B220-APC (RA3-6B2) from $\mathrm{BD}$ Biosciences (San Jose, CA), and JAML-PE (4E10; Biolegend, San Diego, CA). Mouse-Alexa488 and rabbit-Alexa555 (Invitrogen), rabbit anti-Phospho-cRaf and Phospho-p44/42 MAPK (Erk 1/2) (Cell Signaling, Danvers, MA), mouse-HRP IgG, and goat anti-rabbit Ig $\gamma$ (Jackson Immunoresearch, West Grove, PA). HRP-conjugated antiGST (GE Healthcare Biosciences, Pittsburgh, PA), murine anti-His (Abcam, Cambridge, MA), and HRP-conjugated Streptavidin (Zymed, San Francisco, CA). Antibodies were biotinylated using Ezlink Sulfo-NHS-LC-Biotin (Thermo Scientific, Rockford, IL) or conjugated to $\mathrm{CNBr}$-Sepharose 4 Fast Flow beads (GE Healthcare) according to the manufacturer's instructions. ABTS (2,2'-azinobis- (3-ethylbenzothiazoline-6-sulfonic acid), phenanthroline monohydrate, $\mathrm{N}$-Ethylmaleimide, phenylmethylsulfonyl fluoride, protease inhibitor cocktail (\#P8340), phenylmethylsulfonyl fluoride, PMA, Hank's balanced salt solution with/without $\mathrm{Ca}^{2+}$ and $\mathrm{Mg}^{2+}\left(\mathrm{H}^{+} / \mathrm{H}^{-}\right)$, fMLF and phosphatase inhibitors cocktails 1 and 2 were from Sigma (St Louis, MO). TAPI-2 obtained from Calbiochem (Merck, Darmstadt, Germany). 
Surface biotinylation, immunoprecipitation, and immunoblotting. HL60, PLB-985 cells, or PMN were washed, resuspended in $\mathrm{H}^{-}$at $5 \times 10^{7}$ per $\mathrm{ml}$ and incubated with $8 \mathrm{mg}$ Ezlink Sulfo-NHS-LC-Biotin dissolved in $1 \mathrm{ml}$ PBS $\left(30 \mathrm{~min} 4{ }^{\circ} \mathrm{C}\right)$. After quenching in $20 \mathrm{ml} 40 \mathrm{~mm}$ Tris- $\mathrm{HCl} \mathrm{pH} \mathrm{8.0,100} \mathrm{mM} \mathrm{NaCl,} \mathrm{cell} \mathrm{lysates} \mathrm{were} \mathrm{prepared} \mathrm{for}$ immunoprecipitation and immunoblotting as described. ${ }^{6}$

Detection of soluble JAML by capture ELISA. Immulon II microtiter plates were coated with mAb DW100 $\left(1 \mu\right.$ g per well, overnight at $\left.4{ }^{\circ} \mathrm{C}\right)$, then blocked with 5\% Roche blocking reagent and incubated with supernatants of stimulated PMN, HL60, or PLB-985 as well as serial dilutions of purified sJAML $(100 \mu \mathrm{l}, 1 \mathrm{~h}$, room temperature). After washing, biotinylated DW216 $\left(10 \mu \mathrm{g} \mathrm{ml}^{-1}\right)$ was added followed by incubation with streptavidin-HRP. Binding was analyzed in a microplate reader at $405 \mathrm{~nm}$ after addition of ABTS.

CAR binding assay. Immulon II plates were coated with sJAML $\left(10 \mu \mathrm{g} \mathrm{ml}^{-1}\right.$ in PBS, overnight at $\left.4^{\circ} \mathrm{C}\right)$ and blocked as described above. CAR-GST $\left(10 \mu \mathrm{g} \mathrm{ml}^{-1}\right.$ in $100 \mu \mathrm{l}, 1 \mathrm{~h}$ room temperature) was added to wells in the presence of antibody solutions $(10 \mu \mathrm{l}, 1 \mathrm{~h}$, room temperature) followed by goat anti-GST-HRP and ABTS as above.

Cell isolation and flow cytometric analysis. Isolation of blood and lamina propria cells was performed as previously described. ${ }^{47}$ Following Fc receptors block ( $\alpha$-Fc $\gamma$ RIII/II, 2.4G2) cells were prepared for flow cytometry and analyzed using LSR II Cytometer. CD $45^{+}$live cells were gated and cell populations were defined as follows: $\mathrm{CD} 3 \epsilon^{+} \mathrm{TCR} \gamma \delta^{+}$cells $\left(\gamma \delta \mathrm{T}\right.$ cells), $\mathrm{Grl}^{+} \mathrm{B}_{220^{-}}$(neutrophils), and $\mathrm{Grl}^{-} \mathrm{B} 220^{+}$(B cells). To analyze JAML expression on PMN adherent to T84 epithelial cell, PMN $\left(2.5 \times 10^{5}\right.$ cells per well) were applied to T84 monolayers $(15 \mathrm{~min})$, harvested with trypsin/EDTA and stained as previously described, ${ }^{9}$ gating on CD18/CD11b-positive cells. $\triangle \mathrm{MFI}$ represents the mean fluorescence intensity minus the mean fluorescence intensity of $\operatorname{IgG}$ control.

Cell adhesion assay. Intestinal epithelial cells (T84/Caco-2) were seeded on 24 -well plates $\left(1 \times 10^{6}\right.$ cells per well $)$ and cultured to confluency. PMN or HL60 cells were loaded with CellTracker Green (CMFDA, Life Technologies, Grand Island, NY, $10 \mathrm{~min}$ at $37^{\circ} \mathrm{C}$ ) and added to wells $\left(2.5 \times 10^{5}\right.$ cells per well) for $1 \mathrm{~h}$ (in the absence of extracellular $\mathrm{Ca}^{2+}$, to open up junctions) at $37^{\circ} \mathrm{C}$. After washing, adherent PMN were lysed with $1 \%$ Triton X-100. Fluorescence intensity was measured using FluoStar Galaxy plate reader (BMG Labtechnologies Inc., Durham, NC) at excitation/emission wavelengths of $485 / 535 \mathrm{~nm}$. Data are presented as relative fluorescence intensity minus background fluorescence of epithelial monolayer alone.

Immunofluorescence microscopy. PMN and epithelial cells were immunofluorescently stained using standard protocols as previously described. ${ }^{27}$ All images were acquired on a LSM 510 confocal microscope (Carl Zeiss, Thornwood, NY) with Plan-Neofluor $\times 60$ and $\times 40$ objectives.

Assessment of cell proliferation and apoptosis. Epithelial cell proliferation and apoptosis were assessed using the Click-iT EdU Alexa 488 cell proliferation kit (Invitrogen) and Tunel staining (In Situ Cell Death Detection Kit; Roche, Mannheim, Germany), respectively. Monolayers were incubated for $24 \mathrm{~h}$ at $37^{\circ} \mathrm{C}$ before addition of EdU or In Situ Cell Death Detection kits. Five random fields per condition were analyzed and data presented as \% proliferating/apoptotic cells in each field.

Epithelial wound closure assays. Wounds were introduced to confluent T84 and Caco-2 monolayers and monitored as previously described. ${ }^{48}$ Colonoscopy in live mice: Superficial wounds were introduced in vivo (mouse colon) using colonoscopic biopsy techniques as described. ${ }^{26}$ PBS $(100 \mu \mathrm{l})$, Ad5 $(10 \mu \mathrm{g}$ per $100 \mu \mathrm{l})$, Ad11 $(10 \mu \mathrm{g}$ per $100 \mu \mathrm{l})$, or sJAML $(20 \mu \mathrm{g}$ per $100 \mu \mathrm{l})$ was administered intraperitoneally twice daily. All procedures were approved by the Emory University IACUC and performed according to NIH criteria.
Dextran sodium sulfate treatment. Dextran sodium sulfate (MP Biomedicals, Santa Ana, CA) was given to mice in drinking water at a concentration of $3 \%(\mathrm{wt} / \mathrm{vol})$ for 5 days and disease activity was assessed as previously described. ${ }^{49}$

Statistics. Statistical significance was assessed by a Student $t$-test or by one-way ANOVA with a Newman-Keuls Multiple Comparison Test using GraphPad Prism (V4.0, GraphPad Software Inc., La Jolla, CA), and set at $P<0.05$.

SUPPLEMENTARY MATERIAL is linked to the online version of the paper at http://www.nature.com/mi

\section{ACKNOWLEDGMENTS}

We thank Emory DDRDC core facility technical support in culturing intestinal epithelial cell lines (supported by funding from NIH DK064399). We also thank Dr Laur and the Custom Cloning Center Facility at Emory University for the generation of JAML constructs. This work was supported in part by grants from the NIH (DK072564, DK061379, DK079392, DK055679, and DK059888), trainee support from the NIH (DK007771), Career Development Award from the CCFA to Dr Sumagin (CCFA3597).

\section{DISCLOSURE}

The authors declared no conflict of interest.

c) 2014 Society for Mucosal Immunology

\section{REFERENCES}

1. Rhee, S.H., Keates, A.C., Moyer, M.P. \& Pothoulakis, C. MEK is a key modulator for TLR5-induced interleukin-8 and MIP3 alpha gene expression in non-transformed human colonic epithelial cells. J. Biol. Chem. 279, 25179-25188 (2004).

2. MacDermott, R.P., Sanderson, I.R. \& Reinecker, H.C. The central role of chemokines (chemotactic cytokines) in the immunopathogenesis of ulcerative colitis and Crohn's disease. Inflamm. Bowel. Dis. 4, 54-67 (1998).

3. Mrsny, R.J. et al. Identification of hepoxilin A3 in inflammatory events: a required role in neutrophil migration across intestinal epithelia. Proc. Natl. Acad. Sci. USA 101, 7421-7426 (2004).

4. Nourshargh, S., Krombach, F. \& Dejana, E. The role of JAM-A and PECAM1 in modulating leukocyte infiltration in inflamed and ischemic tissues. J. Leukoc. Biol. 80, 714-718 (2006).

5. Laukoetter, M.G. et al. JAM-A regulates permeability and inflammation in the intestine in vivo. J. Exp. Med. 204, 3067-3076 (2007).

6. Nava, P. et al. JAM-A regulates epithelial proliferation through Akt/betacatenin signalling. EMBO Rep. 12, 314-320 (2011).

7. Severson, E.A. et al. Cis-dimerization mediates function of junctional adhesion molecule A. Mol. Biol. Cell 19, 1862-1872 (2008).

8. Moog-Lutz, C. et al. JAML, a novel protein with characteristics of a junctional adhesion molecule, is induced during differentiation of myeloid leukemia cells. Blood 102, 3371-3378 (2003).

9. Zen, K. et al. Neutrophil migration across tight junctions is mediated by adhesive interactions between epithelial coxsackie and adenovirus receptor and a junctional adhesion molecule-like protein on neutrophils. Mol. Biol. Cell 16, 2694-2703 (2005).

10. Raschperger, E. et al. The coxsackie- and adenovirus receptor (CAR) is an in vivo marker for epithelial tight junctions, with a potential role in regulating permeability and tissue homeostasis. Exp. Cell. Res. 312, 15661580 (2006).

11. Cohen, C.J. et al. The coxsackievirus and adenovirus receptor is a transmembrane component of the tight junction. Proc. Natl. Acad. Sci. USA 98, 15191-15196 (2001).

12. Verdino, P., Witherden, D.A., Havran, W.L. \& Wilson, I.A. The molecular interaction of CAR and JAML recruits the central cell signal transducer PI3K. Science 329, 1210-1214 (2010).

13. Luissint, A.C., Lutz, P.G., Calderwood, D.A., Couraud, P.O. \& Bourdoulous, S. JAM-L-mediated leukocyte adhesion to endothelial cells 
is regulated in cis by alpha4beta1 integrin activation. J. Cell Biol. 183 , 1159-1173 (2008).

14. Witherden, D.A. et al. The junctional adhesion molecule JAML is a costimulatory receptor for epithelial gammadelta Tcell activation. Science 329, 1205-1210 (2010).

15. Eken, C. et al. Polymorphonuclear neutrophil-derived ectosomes interfere with the maturation of monocyte-derived dendritic cells. J. Immunol. 180 817-824 (2008)

16. Ludeman, M.J., Zheng, Y.W., Ishii, K. \& Coughlin, S.R. Regulated shedding of PAR1 N-terminal exodomain from endothelial cells. J. Biol. Chem. 279 18592-18599 (2004).

17. Le Gall, S.M., Auger, R., Dreux, C. \& Mauduit, P. Regulated cell surface proEGF ectodomain shedding is a zinc metalloprotease-dependent process. J. Biol. Chem. 278, 45255-45268 (2003).

18. Koizumi, J.I. et al. Protein kinase $\mathrm{C}$ enhances tight junction barrier function of human nasal epithelial cells in primary culture by transcriptional regulation. Mol. Pharmacol. 74, 432-442 (2008).

19. Ivanov, A.I. et al. Microtubules regulate disassembly of epithelial apical junctions. BMC Cell Biol. 7, 12 (2006).

20. Lee, W.Y. et al. Novel structural determinants on SIRP alpha that mediate binding to CD47. J. Immunol. 179, 7741-7750 (2007).

21. Hopkins, A.M. et al. Organized migration of epithelial cells requires control of adhesion and protrusion through Rho kinase effectors. Am J PhysiolGastrointest Liver Physiol 292, G806-G817 (2007).

22. Salic, A. \& Mitchison, T.J. A chemical method for fast and sensitive detection of DNA synthesis in vivo. Proc. Natl. Acad. Sci. USA 105, 2415-2420 (2008).

23. $\mathrm{Si}, \mathrm{H}$. et al. RNAi-mediated knockdown of ERK1/2 inhibits cell proliferation and invasion and increases chemosensitivity to cisplatin in human osteosarcoma U2-OS cells in vitro. Int. J. Oncol. 40, 1291-1297 (2012).

24. Bergelson, J.M. et al. Isolation of a common receptor for Coxsackie B viruses and adenoviruses 2 and 5. Science 275, 1320-1323 (1997).

25. Kirby, I. et al. Identification of contact residues and definition of the CARbinding site of adenovirus type 5 fiber protein. J. Virol. 74, 2804-2813 (2000).

26. Becker, C., Fantini, M.C. \& Neurath, M.F. High resolution colonoscopy in live mice. Nat. Protoc. 1, 2900-2904 (2006).

27. Chin, A.C., Lee, W.Y., Nusrat, A., Vergnolle, N. \& Parkos, C.A. Neutrophilmediated activation of epithelial protease-activated receptors-1 and-2 regulates barrier function and transepithelial migration. J. Immunol. 181, 5702-5710 (2008).

28. Mayerle, J. et al. Extracellular cleavage of E-cadherin by leukocyte elastase during acute experimental pancreatitis in rats. Gastroenterology 129, 1251-1267 (2005).

29. Zemans, R.L. et al. Neutrophil transmigration triggers repair of the lung epithelium via beta-catenin signaling. Proc. Natl. Acad. Sci. USA 108 , 15990-15995 (2011).

30. Rochat, T., Casale, J., Hunninghake, G.W. \& Peterson, M.W. Neutrophil cathepsin $\mathrm{G}$ increases permeability of cultured type II pneumocytes. Am. J. Physiol. 255, C603-C611 (1988).

31. Nusrat, A., Parkos, C.A., Liang, T.W., Carnes, D.K. \& Madara, J.L. Neutrophil migration across model intestinal epithelia: monolayer disruption and subsequent events in epithelial repair. Gastroenterology 113 , 1489-1500 (1997).

32. Li, Y., Brazzell, J., Herrera, A. \& Walcheck, B. ADAM17 deficiency by mature neutrophils has differential effects on L-selectin shedding. Blood 108, 2275-2279 (2006).
33. Hafezi-Moghadam, A. \& Ley, K. Relevance of L-selectin shedding for leukocyte rolling in vivo. J. Exp. Med. 189, 939-948 (1999).

34. Carson, S.D., Hobbs, J.T., Tracy, S.M. \& Chapman, N.M. Expression of the coxsackievirus and adenovirus receptor in cultured human umbilical vein endothelial cells: regulation in response to cell density. J. Virol. 73, 7077-7079 (1999).

35. Vigl, B. et al. Coxsackie- and adenovirus receptor (CAR) is expressed in lymphatic vessels in human skin and affects lymphatic endothelial cell function in vitro. Exp. Cell. Res. 315, 336-347 (2009).

36. Excoffon, K.J.D.A., Hruska-Hageman, A., Klotz, M., Traver, G.L. \& Zabner, $\mathrm{J}$. A role for the PDZ-binding domain of the coxsackie B virus and adenovirus receptor (CAR) in cell adhesion and growth. J. Cell Sci. 117, 4401-4409 (2004).

37. Leca, G., Mansur, S.E. \& Bensussan, A. Expression of VCAM-1 (CD106) by a subset of TCR gamma delta-bearing lymphocyte clones. Involvement of a metalloprotease in the specific hydrolytic release of the soluble isoform. $\mathrm{J}$. Immunol. 154, 1069-1077 (1995).

38. Severson, E.A. \& Parkos, C.A. Structural determinants of Junctional Adhesion Molecule A (JAM-A) function and mechanisms of intracellular signaling. Curr. Opin. Cell Biol. 21, 701-707 (2009).

39. Mandell, K.J., Babbin, B.A., Nusrat, A. \& Parkos, C.A. Junctional adhesion molecule 1 regulates epithelial cell morphology through effects on beta 1 Integrins and Rap1 activity. J. Biol. Chem. 280, 11665-11674 (2005).

40. Kirby, I. et al. Adenovirus type 9 fiber knob binds to the coxsackie B virus-adenovirus receptor (CAR) with lower affinity than fiber knobs of other CAR-binding adenovirus serotypes. J. Virol. 75, 7210-7214 (2001).

41. Patzke, C. et al. The coxsackievirus-adenovirus receptor reveals complex homophilic and heterophilic interactions on neural cells. J. Neurosci. 30, 2897-2910 (2010).

42. van Raaii, M.J., Chouin, E., van der Zandt, H., Bergelson, J.M. \& Cusack, S. Dimeric structure of the coxsackievirus and adenovirus receptor D1 domain at 1.7 A resolution. Structure. 8, 1147-1155 (2000).

43. Volk, A.P. et al. Priming of neutrophils and differentiated PLB-985 cells by pathophysiological concentrations of TNF-alpha is partially oxygen dependent. J. Innate Immun. 3, 298-314 (2011).

44. Tucker, K.A., Lilly, M.B., Heck, L. Jr. \& Rado, T.A. Characterization of a new human diploid myeloid leukemia cell line (PLB-985) with granulocytic and monocytic differentiating capacity. Blood 70, 372-378 (1987).

45. Parkos, C.A., Delp, C., Arnaout, M.A. \& Madara, J.L. Neutrophil migration across a cultured intestinal epithelium. Dependence on a CD11b/CD18mediated event and enhanced efficiency in physiological direction. J. Clin. Invest. 88, 1605-1612 (1991).

46. Balsam, L.B., Liang, T.W. \& Parkos, C.A. Functional mapping of CD11b/ CD18 epitopes important in neutrophil-epithelial interactions: a central role of the I domain. J. Immunol. 160, 5058-5065 (1998).

47. Denning, T.L. et al. Functional specializations of intestinal dendritic cell and macrophage subsets that control Th17 and regulatory Tcell responses are dependent on the T cell/APC ratio, source of mouse strain, and regional localization. J. Immunol. 187, 733-747 (2011).

48. Hopkins, A.M. et al. Epithelial cell spreading induced by hepatocyte growth factor influences paxillin protein synthesis and posttranslational modification. Am. J. Physiol. Gastrointest. Liver Physiol. 287, G886-G898 (2004).

49. Khounlotham, M. et al. Compromised intestinal epithelial barrier induces adaptive immune compensation that protects from colitis. Immunity $\mathbf{3 7}$, 563-573 (2012). 